\author{
Cristiane Soares Leal ${ }^{1}$ \\ Dinaiara Valéria da Silva ${ }^{1}$ \\ Maria da Conceição Coutinho da Silva ${ }^{1}$ \\ Karinn de Araujo Soares Bastos ${ }^{2}$ \\ Disraeli Reis da Rocha Filho ${ }^{1}$
}

Artigo original

\section{Avaliação epidemiológica dos pacientes diabetes mellitus e hipertensão arterial}

Epidemiological evaluation of patients with diabetes mellitus and arterial hipertension

\begin{abstract}
A B S T R A C T
The Hiperdia Program is configured as a registration and follow-up of users and bearers of Diabetes Mellitus and Hypertension in the family health program and aims to monitor patients and generate information for acquisition, dispensation and distribution of medications on a regular basis. The objective of this study was to analyze the socioeconomic, demographic and epidemiological profile of diabetic and hypertensive patients attended by a Family Health team at the Anita Ferraz Health Unit in Teresina-PI. The study was carried out taking into account the objectives of the Unified Health System (SUS), the National Primary Care Policy, the Family Health Strategy and the Hiperdia Program Organization, and the sample consisted of 110 patients. It was found that the majority of hypertensive and diabetic patients were between 40 and 60 years of age, $78.1 \%$ were hypertensive and $58.8 \%$ stated that they had been diagnosed with hypertension and / or diabetes before the age of 45 years. Regarding the profile of hypertensive and diabetic patients, it was observed that, among the risk factors described in the literature, some drew attention, highlighting the non-performance of physical activity, the use of fat, predominantly soybean oil, and excessive consumption of white sugar. Such habits and patterns of consumption, however, can harm the health of these patients, making them susceptible to clinical complications. Based on the results obtained in the study, it was concluded that the involvement of the Family Health Team in hypertensive and diabetic patients in relation to health education is indispensable.
\end{abstract}

\section{R E S U M O}

O Programa Hiperdia configura-se como o cadastramento e acompanhamento dos usuários portadores de Diabetes Mellitus e Hipertensão Arterial, no programa de saúde da família, e tem por objetivo monitorar os pacientes e gerar informação para aquisição, dispensação e distribuição de medicamentos de forma regular. O objetivo desta pesquisa foi analisar o perfil socioeconômico, demográfico e epidemiológico dos pacientes diabéticos e hipertensos atendidos por uma equipe de Saúde da Família na Unidade de Saúde Anita Ferraz, em Teresina-PI. O trabalho foi realizado levandose em consideração os objetivos do Sistema Único de Saúde (SUS), da Política Nacional de Atenção Básica, da Estratégia Saúde da Família e da Organização do Programa Hiperdia e a amostra contou com 110 pacientes. Constatou-se que a maioria dos pacientes hipertensos e diabéticos estava na faixa etária entre 40 a 60 anos, $78,1 \%$ são hipertensos e $58,8 \%$ afirmaram ter sido diagnosticado com hipertensão e/ou diabetes antes dos 45 anos. Em relação ao perfil dos pacientes hipertensos e diabéticos, foi observado que, dentre os fatores de risco descritos na literatura, alguns chamaram a atenção, destacando-se a não realização de atividade física, a utilização de gordura, predominando o óleo de soja, e o consumo excessivo de açúcar branco. Tais hábitos e padrões de consumo, no entanto, podem trazer prejuízos à saúde desses pacientes, tornando-os susceptíveis a complicações clínicas. A partir dos resultados obtidos no estudo, concluiu-se que é indispensável o envolvimento da Equipe Saúde da Família junto aos hipertensos e diabéticos com relação à educação em saúde. 


\section{INTRODUÇÃO}

O Sistema Único de Saúde (SUS) é o sistema de saúde brasileiro, que foi definido a partir da Constituição Federal de 1988 e norteia-se por princípios doutrinários e organizativos: a universalidade de atendimento para todos, seja rico ou pobre; a equidade, constituída como uma forma de atendimento que prioriza as necessidades de cada um para que se possa chegar à igualdade, pois cada usuário tem necessidades diferentes e, portanto, todos desejam solução e integralidade da atenção em todos os níveis da assistência (AGUIAR, 2011).

O Programa de Saúde da Família (PSF) foi iniciado pelo Ministério da Saúde que formulou, em 1991, o Programa de Agentes Comunitários de Saúde (PACS) na tentativa de diminuir a mortalidade materno-infantil nas regiões Norte e Nordeste, por meio da expansão para regiões com mais pessoas carentes. No ano de 1993, em dezembro, foi realizado um encontro com os integrantes do Ministério da Saúde sobre o êxito do PACS e a necessidade de incorporar novos profissionais e dar amplitude às ações e à sua forma de abrangência. A reunião foi apoiada pelo Fundo das Nações Unidas para a Infância (UNICEF) e as experiências de outros países com a Atenção Primária auxiliaram para formular o modelo no Brasil (CORDOBA, 2013).

Em 1994, passou a ser divulgado oficialmente o PSF que, de início, é tido como um modelo de assistência à saúde que busca promover ações de promoção e de proteção à saúde do cidadão brasileiro, da família e da comunidade, por meio do trabalho de equipes de saúde determinadas para realizar o atendimento na unidade local de saúde e na comunidade, em nível de atenção básica (AGUIAR, 2011).

Assim, em busca de organizar a assistência ao hipertenso e/ou diabético, o Ministério da Saúde, em 2001, lançou, em todo o país, o Plano de Reorganização da Atenção à Hipertensão Arterial e ao Diabetes, materializado no Programa de Hipertensão Arterial e Diabetes (Hiperdia), um sistema de cadastramento e acompanhamento dos usuários do programa, no qual os profissionais de saúde são incumbidos do atendimento aos pacientes e do preenchimento dos dados. Tem por objetivo monitorar os pacientes e gerar informação para aquisição, dispensação e distribuição dos medicamentos para tratamento dessas condições, de forma regular e organizada (CARVALHO; CLEMENTINO; PINHO, 2008).

Para tanto, o objetivo geral deste estudo foi analisar o perfil socioeconômico, demográfico e epidemiológico de um grupo de pacientes diagnosticados com diabetes mellitus e hipertensão arterial, atendido por uma Equipe de Saúde da Família, na Unidade de Saúde da Família (UBS) do bairro Anita Ferraz, na Cidade de Teresina-Piaui.

\section{MATERIAIS E MÉTODOS}

O estudo foi realizado na Unidade Básica de Saúde(UBS) Anita Ferraz, localizada na zona leste da cidade de Teresina-PI, com atendimento em dois turnos. A Equipe de Saúde da Família, que fez parte deste estudo, foi a equipe do turno da tarde, devido a disponibilidade de horários dos acadêmicos, sendo esta composta por um médico, um enfermeiro, um técnico de enfermagem e cinco agentes comunitários de saúde. A área é composta por sete micro áreas, e duas permanecem descobertas, devido à falta de recursos humanos. A equipe realiza, por demanda espontânea, atendimento ao grupo Hiperdia, puericultura e acompanhamento do pré-natal. $\mathrm{O}$ número de pacientes cadastrados no Hiperdia da equipe é de 130 pacientes e a equipe atende em torno de 4.500 famílias na área descrita.

Os participantes desta pesquisa foram 110 pacientes diagnosticados com diabetes mellitus, hipertensão arterial ou diabetes mellitus/hipertensão arterial, acompanhados pela referida Equipe de Saúde da Família, no período do levantamento de dados (agosto a setembro de 2015).

A amostra foi constituída por todos os pacientes atendidos pela respectiva Equipe de Saúde da Família, no período da coleta de dados, que aceitaram participar do estudo e assinaram um Termo de Consentimento Livre e Esclarecido (TCLE).

Os dados foram coletados por meio de um questionário, contendo 22 questões pertinentes à pesquisa e aos seus objetivos, permitindo uma investigação mais precisa. $\mathrm{O}$ instrumento foi previamente testado, por meio da aplicação de um questionário piloto, com a finalidade de investigar a compreensão das questões pelo paciente e a obtenção de resultados fidedignos.

Esta pesquisa foi aprovada pelo Comitê de Ética em Pesquisa (CEP), da Faculdade Santo Agostinho, com o número CAAE43634315.7.0000.5602. Foram respeitados todos os preceitos éticos contidos na Resolução 466/2012, do Conselho Nacional de Saúde.

\section{RESULTADOS E DISCUSSÃO}

Os dados coletados nesta pesquisa mostraram que $71,6 \%$ da amostra pertencem ao gênero feminino e apenas $28,4 \%$ ao gênero masculino. Os achados relacionados ao gênero do presente estudo corroboram os estudos de Boaventura e Guanoalini (2007), em que a maioria $(81,6 \%)$ pertencia ao gênero feminino, e o de Lima e outros (2011) que mostraram que os pacientes com hipertensão arterial sistêmica $(n=752)$ eram, em sua maioria, do gênero feminino $(69,6 \%)$, as quais estavam organizadas em maior número na faixa etária entre 50 a 59 anos.

De acordo com Lessa e outros (2006), a diferença nos índices de aumento da pressão arterial entre os gêneros (feminino e masculino) pode ter variações, nacionalmente ou internacionalmente, devido aos fatores socioeconômicos, étnicos, de escolaridade, etc. O grande número de mulheres acometidas por hipertensão arterial ocorre devido à modernidade do mundo, em que a mulher se insere cada vez mais rápido no mercado de trabalho, adquirindo, dessa 


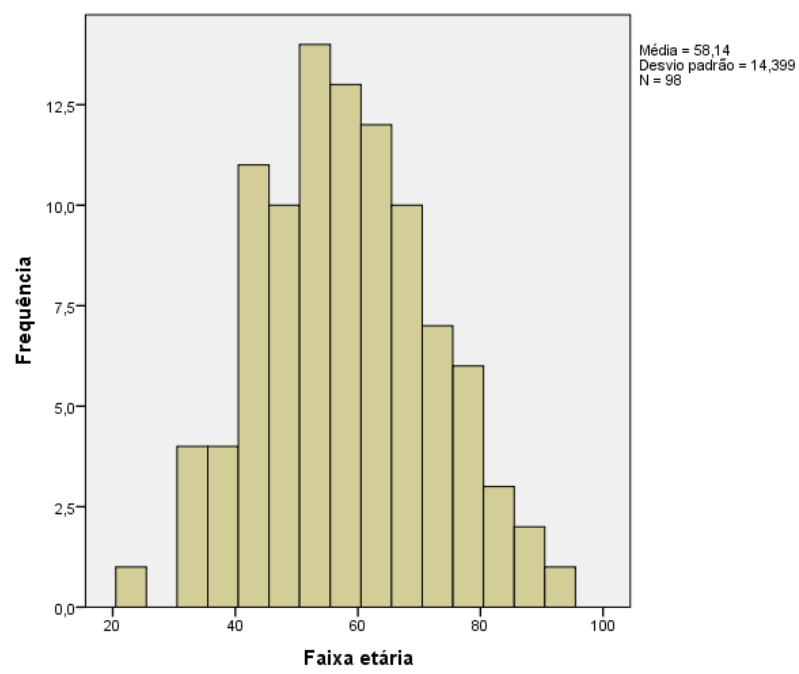

Figura 01. Histograma da idade dos pacientes hipertensos.

\section{Distribuição do gênero}

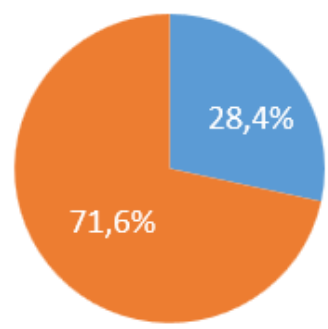

Figura 02. Distribuição do gênero dos pacientes.

maneira, alguns riscos de vida, em consequência, nível de estresse alterado, podendo desencadear diversas patologias e, dentre elas, a hipertensão arterial sistêmica.

Segundo Silva, Oliveira e Pierin (2016), a hipertensão arterial acomete homens e mulheres de maneira semelhante, com prevalência entre os homens até 50 anos de idade. No entanto, a partir da quinta década, há uma predominância maior em mulheres devido às alterações hormonais que ocorrem na menopausa e climatério.

A idade média dos pacientes foi de 58,14 \pm 14,39 anos. Dentre os pacientes que participaram do estudo, a faixa etária que representou maior número de pessoas com hipertensão que procuraram o atendimento da Equipe Saúde da Família (ESF) foi de 40 a 60 anos $(78,1 \%)$.

Diferente dos achados sociodemográficos do presente estudo, Boaventura e Guanoalini (2007), em sua pesquisa, identificaram apenas $25,0 \%$ de sua amostra como portadores de hipertensão arterial.

Como podem ser observados na tabela 1 , os dados desta pesquisa mostraram, ainda, que 64,0\% do grupo Hiperdia que procura atendimento possui a cor parda. Em relação à situação conjugal, os casados $(51,0 \%)$ são os que mais procuram as unidades básicas para atendimento. No que se refere à renda, um percentual considerável $(63,9 \%)$ disse receber apenas um salário mínimo; 20,6\% recebem menos de um salário mínimo e $15,5 \%$ se destacaram por receber de um a três salários mínimos. Quanto ao tipo de gordura mais utilizada na comida, 72,4\% têm preferência pelo óleo virgem. Em se tratando do tipo de açúcar utilizado na alimentação, 65,7\% utilizam o açúcar branco e 33,3\%, o adoçante. Em relação à quantidade de sal utilizada por dia, $76,4 \%$ consomem pelo menos $2,4 \mathrm{~g}$ de sal.

\begin{tabular}{|c|c|c|}
\hline Variáveis & $\mathbf{N}$ & $\%$ \\
\hline \multicolumn{3}{|l|}{ Raça/Cor } \\
\hline Branco & 10 & 10 \\
\hline Pardo & 64 & 64 \\
\hline Negro & 26 & 26 \\
\hline \multicolumn{3}{|l|}{ Conjugal } \\
\hline Casado & 51 & 51 \\
\hline Solteiro & 14 & 14 \\
\hline Viúvo & 22 & 22 \\
\hline Divorciado & 7 & 7 \\
\hline União Estável & 6 & 6 \\
\hline \multicolumn{3}{|l|}{ Renda } \\
\hline < 1 Salário Mínimo & 20 & 20,6 \\
\hline 1 Salário Mínimo & 62 & 63,9 \\
\hline <1-3 Salário Mínimo & 15 & 15,5 \\
\hline \multicolumn{3}{|l|}{ Gordura } \\
\hline Azeite de Oliva & 10 & 10,2 \\
\hline Óleo de soja & 71 & 72,4 \\
\hline Óleo de milho & 1 & 1 \\
\hline Óleo virgem & 8 & 8,2 \\
\hline Outros & 8 & 8,2 \\
\hline \multicolumn{3}{|l|}{ Açúcar } \\
\hline Açúcar Branco & 65 & 65,7 \\
\hline Adoçante & 33 & 33,3 \\
\hline Açúcar Mascavo & 1 & 1 \\
\hline \multicolumn{3}{|l|}{ Sal } \\
\hline $2,4 \mathrm{~g}$ & 42 & 76,4 \\
\hline $3,4 \mathrm{~g}$ & 9 & 16,4 \\
\hline $5 \mathrm{~g}$ & 4 & 7,3 \\
\hline
\end{tabular}

Tabela 01. Relação da distribuição das variáveis: raça e cor, situação conjugal, renda, tipo de gordura, tipo de açúcar e sal.

Os pacientes que se auto referiram de cor parda foram os mais comprometidos pela hipertensão arterial, incluindo os gêneros feminino e masculino, sendo, este dado, compatível com aqueles de Lima e outros. (2011). No que se refere à distribuição de fatores associados à hipertensão arterial sistêmica, em torno de $12,0 \%$ dos entrevistados adicionavam quantidade extra de sal na sua alimentação. Em relação à situação conjugal, 51,0\% dos participantes eram casados no momento da pesquisa.

A tabela 2 mostra que $80,4 \%$ dos hipertensos e diabéticos atendidos e avaliados na UBS afirmaram que não fazem uso de bebida alcoólica. Com relação à atividade física, 80,6\% disseram que não praticavam nenhum tipo de atividade e 
$83,5 \%$, não fazem uso do tabaco. No que se refere à questão de serem portadores de alguma doença, 78,1\% apresentavam hipertensão arterial. Sobre o diagnóstico, 58,8\% responderam que foram diagnosticados com idade menor que 45 anos e 22,7\%, com mais de 50 anos. Ainda, 95,2\% dos pacientes relataram que faziam uso de medicamento.

\begin{tabular}{|c|c|c|}
\hline Variáveis & $\mathrm{N}$ & $\%$ \\
\hline \multicolumn{3}{|l|}{ Ingestão de bebida alcoólica } \\
\hline $1-2$ vezes por semana & 7 & 7,2 \\
\hline $3-5$ vezes por semana & 3 & 3,1 \\
\hline 1 vez por mês & 9 & 9,3 \\
\hline Não faz uso de bebida alcoólica & 78 & 80,4 \\
\hline \multicolumn{3}{|l|}{ Atividade física } \\
\hline 2 vezes na semana & 4 & 4,1 \\
\hline 3 vezes na semana & 7 & 7,1 \\
\hline 4 vezes na semana & 1 & 1 \\
\hline 5 vezes na semana & 7 & 7,1 \\
\hline Nenhuma & 79 & 80,6 \\
\hline \multicolumn{3}{|l|}{ Uso tabaco } \\
\hline 1-2 cigarros & 6 & 6,2 \\
\hline 3-5 cigarros & 3 & 3,1 \\
\hline 6-10 cigarros & 2 & 2,1 \\
\hline 10-20 cigarros & 1 & 1 \\
\hline$>20$ cigarros & 1 & 1 \\
\hline Não faz uso & 81 & 83,5 \\
\hline Uma vez por semana & 3 & 3,1 \\
\hline \multicolumn{3}{|l|}{ Portador doença } \\
\hline Hipertensão Arterial & 75 & 78,1 \\
\hline Diabetes Melitus & 7 & 7,3 \\
\hline $\begin{array}{l}\text { Diabetes Melitus e Hipertensão } \\
\text { Arterial } \\
\text { Diagnóstico }\end{array}$ & 14 & 14,6 \\
\hline$<45$ Anos & 57 & 58,8 \\
\hline $45-50$ & 18 & 18,6 \\
\hline$>50$ & 22 & 22,7 \\
\hline \multicolumn{3}{|l|}{ Tratamento } \\
\hline Medicamentoso & 80 & 95,2 \\
\hline
\end{tabular}

Tabela 02. Distribuição das pessoas com hipertensão e diabetes em relação às variáveis: frequência de ingestão de bebida alcoólica, atividade física, uso do tabaco, tipo de doença diagnosticada, idade do diagnóstico e tipo de tratamento realizado.

Ao contrário desta pesquisa, Costa e outros (2007) encontraram resultados diferentes, uma vez que cerca de $65,0 \%$ da sua amostra ingeriam bebidas alcoólicas em quantidade inferior a 30g/dia e 30,0\% declararam-se fumantes. Por outro lado, a prática de atividade física corrobora os achados de Costa e outros. (2007), mostrando que apenas $20,0 \%$ dos indivíduos de sua pesquisa realizavam atividade física considerada aceitável para atingir benefícios para a saúde.

Santos e outros (2013) observaram que, do total de hipertensos, $72,98 \%$ eram previamente diagnosticados e tomavam medicação para controle da pressão, enquanto que $27,02 \%$ são hipertensos não diagnosticados.

De acordo com tabela 3, o Enalapril é a medicação mais utilizada pelos pacientes $(60,6 \%)$, seguido pela Hidroclorotiazida (59,9\%). Apenas 16,0\% fazem uso de outros medicamentos.

\begin{tabular}{lcc}
\hline \multicolumn{1}{c}{ Medicamentos } & N & $\%$ \\
\hline Hidroclorotiazida & 56 & $59,60 \%$ \\
Enalapril & 57 & $60,60 \%$ \\
Losartana & 9 & $9,60 \%$ \\
Atenolol & 2 & $2,10 \%$ \\
Nifedipino & 4 & $4,30 \%$ \\
Metildopa & 2 & $2,10 \%$ \\
Anlodipino & 4 & $4,30 \%$ \\
Carvedilol & 2 & $2,10 \%$ \\
Propanolol & 10 & $10,60 \%$ \\
Outros & 15 & $16,00 \%$ \\
\hline
\end{tabular}

Tabela 03. Tipo de tratamento medicamentoso utilizado pelos pacientes para o controle da hipertensão e diabetes.

No estudo de Santos et al. (2013), os medicamentos mais utilizados foram os Inibidores da Enzima Conversora de Angiotensina (IECA) associados a diuréticos $(22,92 \%)$ e a monoterapia (18,75\%), o que corrobora o presente estudo.

De acordo com a tabela 4, as medicações encontram-se disponíveis em 75,0\% dos casos, o que corrobora o estudo de Souza, Gomes e outros. (2015) que afirmaram acesso à medicação por parte de $88,0 \%$ dos pacientes. Em relação às necessidades do grupo, 43,9\% disseram ter a necessidade de mais atendimento médico. Segundo Medeiros (2010), com relação ao total de consultas ofertadas, 43,4 \% dos usuários disseram estar satisfeitos; todavia os autores revelaram que a demanda é muito grande para uma minoria de profissionais. No que tange à dificuldade do atendimento, $52,0 \%$ afirmaram que há dificuldade em marcar horário.

No que diz respeito ao tempo de espera, 45,0\% afirmaram que esperam 30 minutos para serem atendidos. De modo semelhante, Souza, Gomes e outros. (2015) afirmaram que o tempo de espera no dia da consulta médica foi de 15 a 30 minutos para 42,0\% dos pacientes atendidos. Com relação à importância da avaliação do grupo de hiperdia, 45,0\% afirmaram que traz qualidade de vida e diminuição dos fatores de risco da hipertensão e diabetes. De acordo com Felbere e outros. (2011), promover a saúde é a ideia central do grupo, e esta ação traz melhoria na qualidade de vida e minimiza as comorbidades e os riscos à saúde, mudando a vida dessas pessoas por meio do incentivo ao convívio social, da melhoria nos hábitos de vida e do acesso ao conhecimento, quanto às doenças instaladas.

Quanto à compra, ou não, da medicação que não está disponível nas UBS, de acordo com sua renda mensal (tabela 5), dos pacientes que utilizam a Hidroclorotiazida, 53,8\% 
Leal, C.S. et al.

\begin{tabular}{|c|c|c|}
\hline Variáveis & $\mathbf{N}$ & $\%$ \\
\hline \multicolumn{3}{|l|}{ Medicações disponíveis } \\
\hline Não & 23 & 23,7 \\
\hline Sim & 75 & 76,5 \\
\hline \multicolumn{3}{|l|}{ Necessidade do grupo } \\
\hline Situação precária da estrutura física & 23 & 23,5 \\
\hline Atendimento de enfermagem & 11 & 11,2 \\
\hline Atendimento médico & 43 & 43,9 \\
\hline Falta de medicamentos & 21 & 21,4 \\
\hline \multicolumn{3}{|l|}{ Dificuldade do atendimento } \\
\hline $\begin{array}{l}\text { Dificuldade de acesso para chegar à } \\
\text { UBS }\end{array}$ & 29 & 29,6 \\
\hline Dificuldade em marcar horário & 51 & 52,0 \\
\hline $\begin{array}{l}\text { Dificuldade em encontrar } \\
\text { funcionário atendendo }\end{array}$ & 18 & 18,4 \\
\hline \multicolumn{3}{|l|}{ Tempo de espera } \\
\hline 10 minutos & 4 & 4,1 \\
\hline 30 minutos & 45 & 45,9 \\
\hline 60 minutos & 10 & 10,2 \\
\hline De 1 a 2 horas & 36 & 36,7 \\
\hline$>$ que 2 horas & 3 & 3,1 \\
\hline \multicolumn{3}{|l|}{ Avaliação do grupo } \\
\hline Nenhuma & 2 & 2,0 \\
\hline Qualidade de vida & 45 & 45,9 \\
\hline $\begin{array}{l}\text { Diminuição dos fatores hipertensão } \\
\text { e diabetes }\end{array}$ & 45 & 45,9 \\
\hline $\begin{array}{l}\text { Atenção de qualidade prestada pela } \\
\text { UBS }\end{array}$ & 6 & 6,1 \\
\hline
\end{tabular}

Tabela 04. Distribuição das variáveis: medicações disponíveis, necessidade do grupo, dificuldade de atendimento, tempo de espera, avaliação do grupo.

afirmaram que a sua renda não é suficiente para a compra da medicação, enquanto que $48,7 \%$ responderam também insuficiência financeira para a compra do Enalapril e 28,2\% relataram essa dificuldade com outros tipos de medicação.

Segundo as V Diretrizes Brasileiras de Hipertensão Arterial (2006), os medicamentos anti-hipertensivos são os mais utilizados, juntamente com os diuréticos tiazídicos, em baixas doses. Os diuréticos de alça são indicados para os casos de hipertensão associada à insuficiência renal, ou insuficiência cardíaca com retenção de líquido. Os inibidores de ECA são indicados para os pacientes com hipertensão que apresentam insuficiência cardíaca, infarto agudo do miocárdio e alto risco para doença aterosclerótica e para os diabéticos, pois retardam o declínio da função renal em pacientes com nefropatia diabética.

\section{CONCLUSÃO}

O presente trabalho possibilitou conhecer o perfil do grupo de hipertensos e diabéticos quanto à situação socioeconômica, demográfica e epidemiológica. Constatouse que a maioria dos pacientes era hipertensa e diabética, e pertencia à faixa etária entre 40 a 60 anos.

\begin{tabular}{lcc}
\hline \multicolumn{1}{c}{ Medicação } & $\mathrm{N}$ & $\%$ \\
\hline Metformina & 4 & $10,3 \%$ \\
Insulina & 2 & $5,1 \%$ \\
Hidroclorotiazida & 21 & $53,8 \%$ \\
Enalapril & 19 & $48,7 \%$ \\
Losartana & 2 & $5,1 \%$ \\
Atenolol & 1 & $2,6 \%$ \\
Nifedipino & 1 & $2,6 \%$ \\
Metildopa & 2 & $5,1 \%$ \\
Anlodipino & 1 & $2,6 \%$ \\
Propanolol & 6 & $15,4 \%$ \\
Outros & 11 & $28,2 \%$ \\
\hline
\end{tabular}

Tabela 05. Dados referentes à compra ou não da medicação que não está disponível nas UBS, de acordo com sua renda mensal.

Em relação ao perfil dos pacientes hipertensos e diabéticos que fazem parte do grupo do Hiperdia desta Estratégia Saúde da Família, chamou a atenção a não realização de atividade física e a utilização de gordura, predominando o óleo de soja, e o maior consumo de açúcar branco, que são prejudiciais e podem piorar a saúde desses pacientes.

Medidas educativas são fundamentais na sensibilização e conscientização para a adesão ao tratamento medicamentoso e não medicamentoso, incluindo a aceitação quanto às orientações oferecidas pelos profissionais da saúde, sobretudo, a participação no Programa Hiperdia (Hipertensos e Diabéticos), que oferece atividades que proporcionam uma melhor qualidade de vida aos pacientes hipertensos e diabéticos.

Portanto, há necessidade de maior investimento da equipe da Estratégia Saúde da Família em educação para a saúde, com a finalidade de evitar o desenvolvimento de complicações frequentes na hipertensão e no diabetes.

\section{REFERÊNCIAS}

AGUIAR, Z. N.SUS: Sistema Único de Saúde: antecedentes, percurso, perspectiva e desafios. São Paulo: Martinari, 2011. 189p.

BOAVENTURA, A. G.; GUANOALINI, R. V. Prevalência de hipertensão arterial e presença de excesso de peso em pacientes atendidos em um ambulatório universitário de nutrição da cidade de São Carlos. São Paulo. Alim.Nutr. Araraquara, v. 18, n.4, p. 381-385, out/dez. 2007.

CARVALHO, V. L. S.; CLEMENTINO, V. Q.; PINHO, L. M. O. Educação em saúde nas páginas da REBEN no período de 1995 a 2005. R. Bras. Enfermagem, v. 2, n.61, p. 243-248, mar./abr. 2008.

CORDOBA, E. SUS e ESF: Sistema Único de Saúde e estratégia saúde da família. São Paulo:Rideal, 2013.

COSTA, D. S. J.; BARCELLOS, F. C.; SCLOWITZ, M. L.et al. Prevalência de hipertensão arterial em adultos e fatores associados:um estudo de base populacional urbana em Pelotas, Rio Grande do Sul, Brasil. Sociedade Brasileira de Cardiologia. Arq. Bras. Cardiol., v. 88, n. 1, p. 59-65, 2007. 
FELBER, D. T.; MACHADO, D. F.; NOLIBOS, E. et al. Programa hiperdia: ações de promoção à saúde em usuários hipertensos e diabéticos do PSF. Anais do salão, v.3, n.3,2011. Disponível em:<http//:www.seer.unipampa.edu.br>. Acesso em: 10 nov.2015.

LESSA, I., MAGALHÃES, L.; ARAÚJO, M. J.et al. Arterial hypertension in the adult population of Salvador(BA), Brazil. Arquivos Brasileiros de Cardiologia, 2006.

LIMA, L. M.; SCHWARTZ, E.; MUNIZ, R. M.et al. Perfil dos usuários do Hiperdia de três unidades básicas de saúde do sul do Brasil. R. Gaúcho Enferm. Porto Alegre,v. 32, n. 2, p. 323-329, jun. 2011.

MEDEIROS, F. A.; ARAÚJO, S.; GEORGIA, C. et al. Acolhimento em uma unidade básica de saúde: a satisfação do usuário em foco. R.Salud Pública. v.12, n.3, p.402-413,2010.

SANTOS, A. B. V.; BARRETO, V. P.; OLIVEIRA, S. M.et al. Perfil epidemiológico da hipertensão arterial sistêmica na população de Cajazeiras, Paraíba. R. Bras. em Saúde, v. 17, n. 3, p. 253-262, 2013.

SILVA, S. S B. E; OLIVEIRA, S. F. S. B.; PIERIN, A. M. G.TheO controle da hipertensão arterial em mulheres e homens: uma análise comparativa. Revista da Escola de Enfermagem da USP, v.50, p.50-58, 2016.2 Disponível em:http://www.scielo.br/pdf/reeusp/v50n1/pt_0080-6234-reeusp50-01-0050.pdf. Acesso em: 23: Set. 2017

SOUZA E.; GOMES, L.T.; GRACIANO, M. M.; TERRA, L. H.et al. Avaliação da atenção primária aos hipertensos cadastrados no hiperdia. R. Enferm UFPE. Recife, v. 9, n. 4, p. 7347-7356, abr.2015.

DIRETRIZES Brasileiras de Hipertensão Arterial, 5.Arq. Bras. Cardio. São Paulo. 2006, v. 89, n. 3. Disponível em: <http://dx.doi.org/10.1590/S0066-782X2007001500012>. Acesso em: 23 abr. 2015. 